\title{
An Integrable SIW Phase Shifter in a Partially Magnetized Ferrite LTCC Package
}

\author{
Ahmed Nafe, Student Member, IEEE, and Atif Shamim, Senior Member, IEEE
}

\begin{abstract}
There is a growing need for small size integrable phased antenna arrays for emerging satellite communications on-the-move applications. Traditional ferrite-based phase shifters are generally bulky due to the need of electromagnets for biasing, yielding them unsuitable for this kind of application. In this paper, a novel compact light-weight substrate integrated waveguide (SIW) based phase shifter realized in a multi-layer ferrite low-temperature co-fired ceramic package with embedded bias windings is reported. By using embedded windings and operating the material in a partially magnetized state, the required bias magnetic field could be significantly reduced from typically about 1000 Oe to less than 50 Oe. Moreover, the presented phase shifter has two modes of operations corresponding to two different biasing scenarios of the SIW, namely, symmetric and anti-symmetric bias. Under anti-symmetric bias, the phase shifter can achieve high nonreciprocal phase shift, whereas under symmetric bias, the phase shift is reciprocal, but the available phase shift is less than the anti-symmetric case. The fabricated prototype operates in the 11.5-13.5-GHz range and has a peak figure of merit (phase shift per decibel of loss) of $102^{\circ} / \mathrm{dB}$ and a maximum phase shift per unit length of $153^{\circ} / \mathrm{cm}$, which are more than five times the previously reported figures for this technology. Due to the use of embedded windings, the presented phase shifter offers a huge size reduction from the order of $\mathrm{cm}^{3}$ to $\mathbf{m m}^{3}$, making it particularly useful for mobile phased-array applications.
\end{abstract}

Index Terms-Ferrite phase shifter, low-temperature co-fired ceramic (LTCC), substrate integrated waveguide (SIW).

\section{INTRODUCTION}

$\mathbf{P}$ HASED antenna arrays are widely used in a variety of applications such as imaging and communication systems, as well as different types of radars (automotive, weather, and military). Recently, increasing attention is given to lightweight and small footprint phased antenna arrays suitable for employment in mobile satellite communication-on-the-move user terminals that require smart antennas with controllable radiation direction [1], [2]. Phase shifters are key components in such arrays as they are responsible for the beam-steering function. A phased antenna array can require hundreds or even thousands of phase shifters [3]. It is thus always desirable to find new phase shifters that are more integrable and of smaller size and weight

Manuscript received September 07, 2014; revised January 26, 2015, March 18, 2015, and March 28, 2015; accepted May 01,2015. This work was supported by the King Abdullah University of Science and Technology (KAUST).

The authors are with the Department of Electrical Engineering, King Abdullah University of Science and Technology (KAUST), Thuwal, Jeddah 239556900, Saudi Arabia (e-mail: ahmed.nafe@kaust.edu.sa)

Color versions of one or more of the figures in this paper are available online at http://ieeexplore.ieee.org.

Digital Object Identifier 10.1109/TMTT.2015.2436921

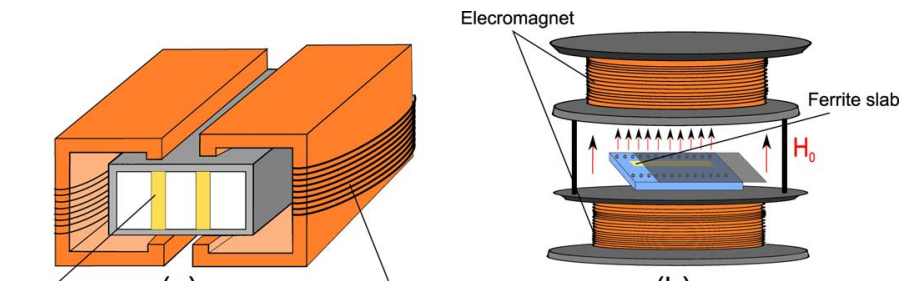

Ferrite Slab

(a)

Windings

(b)

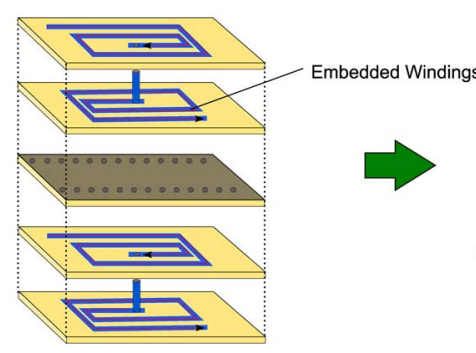

$\square$ Non-magnetic material $\square$ Ferrite material Windings Core material

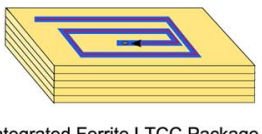

Integrated Ferrite LTCC Package

(c)

Fig. 1. Different ferrite-based phase shifters topologies. (a) Rectangular waveguide with coils for biasing. (b) SIW in a normal machined substrate that has a ferrite slab inserted into it. (c) SIW in ferrite LTCC with embedded bias windings.

in order to reduce the overall cost, obtain better form factor, and increase mobility of phased antenna arrays.

Tunable microwave phase shifters have been implemented in several technologies such as monolithic microwave integrated circuits (MMICs), microelectromechanical systems (MEMS), and varactor diodes, as well as on tunable materials such as ferroelectrics and ferrites[4]. Among these technologies, ferrite waveguide based phased shifters are known to offer special merits in terms of achieving very low insertion loss $(<1 \mathrm{~dB})$, obtaining higher phase shifter figure of merit (FoM), and better power handling relative to competing technologies[2], [4], [5]. However, due to their bulky nonplanar nature and the typical need for fitting of external coils and ferrite yokes to the waveguide, as illustrated in Fig. 1(a), they are nonintegrable and produced as discrete units. For integration in phased antenna arrays, they often require complicated feed assemblies with metallic support structures, which add to the cost and weight of the array [3]. This renders them unsuitable for applications that require mobility such as satellite on-the-move communications.

Over the past decade, the concept of substrate integrated waveguide (SIW) gained increased popularity as an integrated replacement of rectangular waveguides[6]. It combines the advantages of a rectangular waveguide such as low loss and high quality factor while being compatible with planar, low-cost, and integrated microwave circuits [6]. Due to its aforementioned merits, it has been extensively employed to realize a variety 
of microwave components such as filters [7], couplers [8], and antennas [9]. Also, several works have reported combining SIW and bulk microwave ferrite materials, which are cut into slabs and inserted into a nonmagnetic substrate, as depicted in Fig. 1(b) to realize isolators [10], switches[11], circulators [12], and phase shifters [13]. However, this approach suffers from two main drawbacks, which are: 1) the requirement for substrate machining that complicates the fabrication process and 2) the use of bulky coil systems, needed to generate the biasing magnetic field for the ferrite material, which limit the device mobility. Though this approach may be acceptable for developing standalone phase shifters, it is clearly infeasible for producing tens or hundreds of units for application in phased-array systems, especially since a separate electromagnet is required for each unit.

Recently, ferrite material has been introduced in low temperature co-fired ceramic (LTCC) multilayer packaging technology yielding LTCC packages made entirely of ferrite material with printed metal traces and vias. This enabled the realization of ferrite-based components such as tunable antennas [14], [15] and filters [16] in a compact, lightweight, and mass-producible package. Since the biasing circuitry can be embedded in the ferrite package, as demonstrated in Fig. 1(c), this eliminates the need for any bulky coil systems that is usually employed to bias the ferrite material and tune its properties. An additional benefit of embedding the bias windings inside the package is the removal of demagnetization effects ${ }^{1}$ that occur in case of externally applied biasing magnetic field. This enables the properties of the material to be tuned using an internally generated magnetic field, which is about $5 \%$ of the typically required external field [14].

To the authors' best knowledge, there have been only two reported publications on the use of ferrite LTCC for phase-shifter realization. The first is the paper by Stitzer [18], which was mainly concerned about material modeling and presented little information about a planar strip-line phase-shifter prototype. The second is the one by Bray and Roy [19], which presented the theory for a new nonreciprocal SIW phase shifter with high phase-shifting capability. However, the fabricated prototype did not perform well and could not meet the theoretical expectations achieving a measured FoM of about $15^{\circ} / \mathrm{dB}$ and a phase-shift capability of $26^{\circ} / \mathrm{cm}$.

In this work, we demonstrate that a FoM close to $100^{\circ} / \mathrm{dB}$ is achievable in this technology. A dual-operation phase-shifter design that can achieve nonreciprocal, as well as reciprocal operation is developed by utilizing symmetric and anti-symmetric biasing configurations, depicted in Fig. 2, marking the first time a reciprocal ferrite-based phase shifter is realized in SIW technology. The reciprocal property is required for a phase shifter employed in simultaneous transmit/receive antenna arrays [20], [21]. In addition, the device achieves better performance relative to the previously reported one, increasing the FoM by nearly

\footnotetext{
${ }^{1}$ Demagnetization effects refer to the reduction of the external magnetic field $H_{e}$ applied to a ferrite material resulting in establishing an internal magnetic field $H_{i}$ within the material whose value is lower than the applied field. It is governed by $H_{i}=H_{e}-N_{d} M_{s}$, where $N_{d}$ is called the demagnetization factor [17]
}

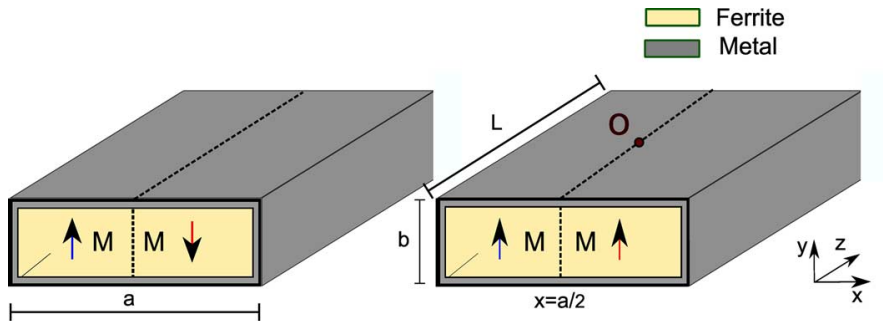

(a)

(b)

Fig. 2. Biased waveguide configurations. (a) Anti-symmetric. (b) Symmetric.

six times, the normalized phase shift by about five times, and reducing required current by $25 \%$.

This paper is organized as follows. Section II introduces the theory and phase-shifting properties of symmetrically and anti-symmetrically biased waveguides in partially magnetized state. In Section III, the simulation model is validated through comparison with the analytical results. In Section IV, design details, simulation model, and results of the developed SIW phase shifter are presented. Fabricated prototype characterization and comparison to simulated performance are discussed in Section $\mathrm{V}$ along with comparison against other ferrite-based phase shifters reported in the literature. In Section VI, the concluding remarks of this work are summarized.

\section{Symmetricly AND ANTI-SYMMETRICLY BIASED WAVEGuides IN PARTIALly Magnetized FERRITES}

Ferrites phase shifters can be broadly classified into two categories. The first category includes phase shifters that operate in saturation and near the ferromagnetic resonance frequency $f_{\mathrm{FMR}}$ such as [22] and [23] and the second category include those operating in the partially magnetized (unsaturated) state such as [24]. Operation near $f_{\mathrm{FMR}}$ will enable large changes in the ferrite permeability tensor to be achieved, and consequently, large phase shifts. However, ferrite materials near $f_{\mathrm{FMR}}$ are very lossy and generally large biasing magnetic fields ( $>1 \mathrm{kOe})$ are required to position the resonance in the gigahertz frequency range $\left(f_{\mathrm{FMR}} \propto H\right)$. On the other hand, operation in a partially magnetized state does not require such high magnetic field for biasing, where the ferrite properties can be dynamically tuned by driving the magnetization state of the material between the unmagnetized state $(M=0)$ and the saturated state $\left(M=M_{s}\right)$, which typically requires low bias fields $(<100 \mathrm{Oe})$ for soft ferrite materials. An important limitation of using unsaturated ferrites is the need to operate above the material's magnetization frequency $\left(f_{m}=2.8(\mathrm{MHz} / \mathrm{G}) \times M_{s}\right)$ to avoid low-field losses [25], [26]. In this work, the phase shifter is designed to be operated in partially magnetized state $\left(M \leq M_{s}\right)$ to minimize the bias field requirements. Section II-A will elaborate on the properties of partially magnetized ferrites and their models.

\section{A. Ferrites in Partially Magnetized State}

Different from saturated ferrite materials, in which all domains magnetizations are aligned and whose properties are mainly dependent on the internal magnetic field $H_{i}$ according to the well-known Polder's tensor [17], partially magnetized ferrites are composed of domains, which are random in shape 


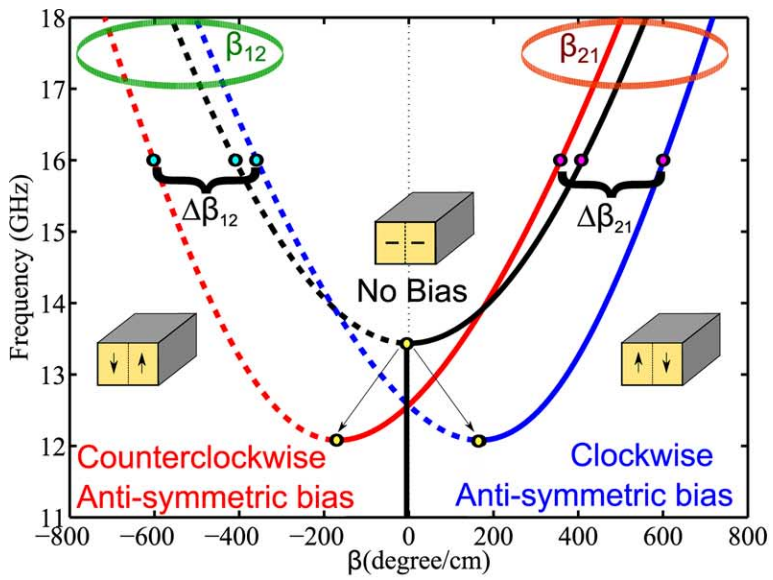

(a)

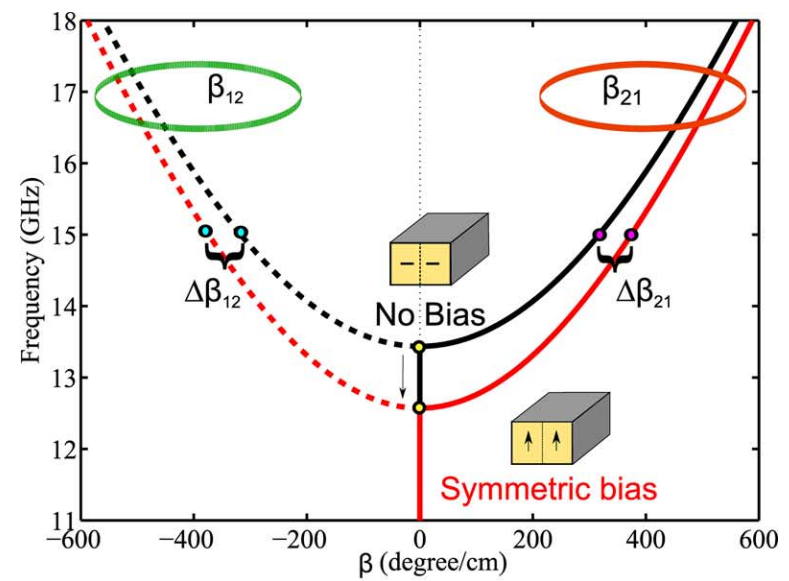

(b)

Fig. 3. Computed dispersion diagram of: (a) an anti-symmetrically and (b) a symmetrically biased waveguide having $a=3.5 \mathrm{~mm}$ and $M_{s}=4000 \mathrm{G}$ with $n_{k}=0.5$ and $\epsilon_{r}=14.5$.

and not fully aligned, but associated with a net magnetization $M$. This leads to complications in modeling the material that always involves spatial averaging [27]. In a completely demagnetized state $(M=0)$, the material is characterized by a scalar relative permeability $\mu_{d}$. Under the assumption of cylindrical domains, Schlömann [28] derived the expression in (1), which was later found to be in good agreement with experimental data

$$
\mu_{d}=\frac{1}{3}+\frac{2}{3} \sqrt{1-\left(\frac{f_{m}}{f}\right)^{2}} .
$$

When the material is magnetized $(M>0)$, the permeability has the form of a gyrotropic tensor similar to Polder's tensor. The tensor permeability for a $y$-biased partially magnetized ferrite is given in (2), diagonal elements are usually expressed using the empirical equations of Green and Sandy [29] given by (3) and (4) as follows:

$$
\begin{aligned}
{[\mu]_{\mathrm{PMS}}^{y-\text { bias }} } & =\mu_{0}\left[\begin{array}{ccc}
\mu & 0 & -j \kappa \\
0 & \hat{\mu} & 0 \\
j \kappa & 0 & \mu
\end{array}\right] \\
\mu & =\mu_{d}+\left(1-\mu_{d}\right)\left(\frac{M}{M_{s}}\right)^{3 / 2} \\
\hat{\mu} & =\mu_{d}\left(1-\left(M / M_{s}\right)^{5 / 2}\right) .
\end{aligned}
$$

The off-diagonal term $\kappa$ is given by (5) [15]. Here, the factor $n_{k}$ is present to account for deviations from the commonly used expression for $\kappa$, known as Rado's formula [30], which is valid when $f \gg f_{m}$ [27]. In this form, the expression could be used to express $\kappa$ for frequencies close to, as well as far above, $f_{m}$,

$$
\kappa=n_{k} \frac{f_{m}}{f} \frac{M}{M_{s}} .
$$

\section{B. Anti-Symmetric Bias}

A ferrite-filled rectangular waveguide biased anti-symmetrically, with oppositely directed bias field (or magnetization) for $x<a / 2$ and $x>a / 2$, as illustrated in Fig. 2(a), has been shown theoretically to possess large phase-shifting capability [19]. In the absence of loss, the dispersion relation for the $\mathrm{TE}_{10}$-like mode is given by (6)[19], where $k_{e}^{2}=\omega^{2} \mu_{e} \epsilon$ is called the extraordinary wavenumber and $\mu_{e}=\left(\mu^{2}-\kappa^{2}\right) / \mu$ is the effective permeability experienced by a wave propagating through a transversely biased ferrite and with the dc bias magnetic field orthogonal to the microwave magnetic field [17],

$$
j \beta \kappa+j \mu \sqrt{k_{e}^{2}-\beta^{2}} \cot \left(\frac{a}{2} \sqrt{k_{e}^{2}-\beta^{2}}\right)=0 .
$$

This equation can be solved for its roots representing the propagation constant $\beta$ for each frequency and magnetization sate. In general there will be two distinct roots representing the two possible propagation directions. A demonstrating example showing the effect of biasing on the dispersion diagram for anti-symmetric bias is illustrated in Fig. 3(a). As can be seen, in the unbiased state, the dispersion diagram is symmetric around the $\beta$ $=0$ axis and the propagation constants in opposite direction $\left(\beta_{21}, \beta_{12}\right)$ are equal in magnitude indicating reciprocal propagation. When the guide is anti-symmetrically biased in a clockwise or counterclockwise sense, the dispersion curve loses its symmetry yielding unequal propagation constants at a given frequency. The maximum phase shift for a given propagation direction in this configuration occurs when the guide is switched between saturation in a clockwise sense to saturation in a counterclockwise sense (indicated on the figure as $\Delta \beta_{21}$ and $\Delta \beta_{12}$ ).

\section{Symmetric Bias}

If a ferrite-filled waveguide is biased symmetrically with respect to its transverse plane ( $x=a / 2)$, as shown in Fig. 2(b), the structure becomes invariant to $180^{\circ}$ rotation about its central transverse axis (passing through point $\mathrm{O}$ ), which would yield reciprocal propagation characteristics [31]. Assuming that $M$ does not vary with position and ignoring losses, the following simple dispersion equation can be obtained assuming a $\mathrm{TE}_{10}$-like mode:

$$
\beta^{2}=k_{e}^{2}-\left(\frac{\pi}{a}\right)^{2} .
$$

For a given frequency (above cutoff) and magnetization state, this dispersion relation always admits two real roots of opposite sign $\left(\beta_{21}=-\beta_{12}\right)$. Fig. 3(b) demonstrates the symmetry of the dispersion diagram with respect to the frequency axis in 


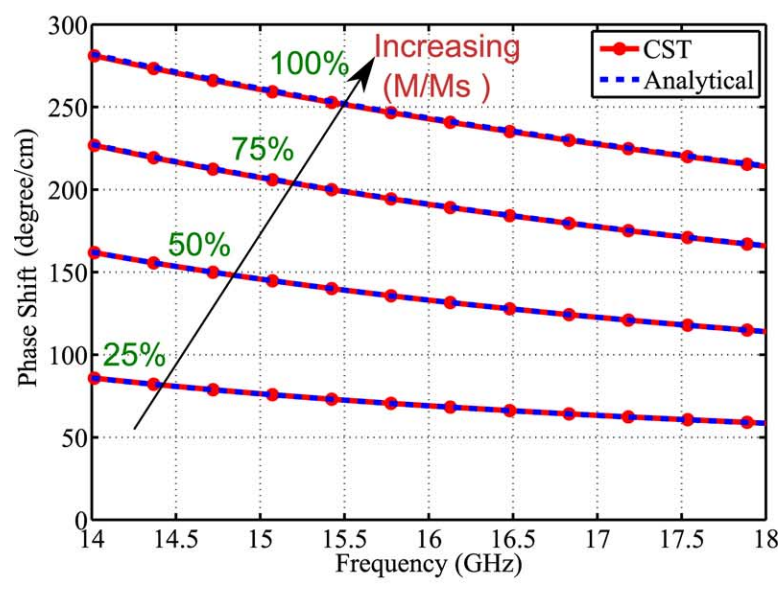

(a)

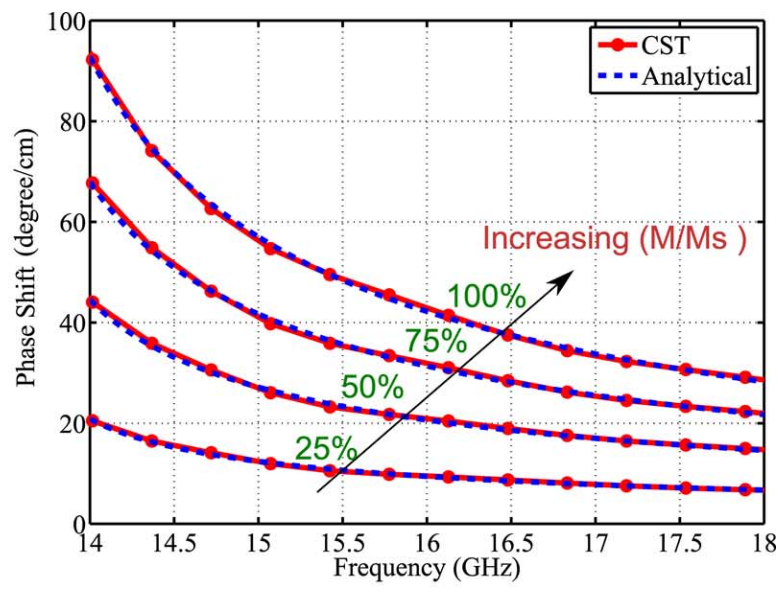

(b)

Fig. 4. Comparison of analytical expressions and CST simulations for the phase shift of: (a) anti-symmetrically and (b) symmetrically biased waveguide having the parameters given in Fig. 3.

the unbiased state as well as in the biased state (in contrast to the anti-symmetric biasing case). Here the maximum reciprocal phase shift occurs when the material is switched between the unbiased and saturated case. The phase shift per centimeter is calculated as $\beta(M)-\beta(0)$ where the subscript for $\beta$ carries no significance in the reciprocal case and thus has been dropped.

\section{Simulation Model VALidation}

As mentioned earlier, here we intend to design the phase shifter with the ferrite material being partially magnetized to minimize the required bias magnetic field. This fact complicates the material modeling in popular commercial simulators, which do not offer such material definition. This problem was solved by using custom full-tensor material definition in CST Microwave Studio. Before going directly into simulating the actual scenario of a SIW with realistic biasing profile generated by actual coils, we first consider a hypothetical idealistic scenario of a rectangular waveguide under uniform symmetric and anti-symmetric magnetization and assuming that no losses are present. The advantage of doing so is that under these conditions the problem could be solved using CST and then directly compared with the solution of analytical equations given in the previous section. This helps to verify the correctness of the simulation results before going into simulating the actual scenario.

The hypothetical waveguide has the same dimensions and material parameter as those stated in the caption of Fig. 3. The structure is then simulated with the ferrite being partially magnetized to different percentages for both symmetric and antisymmetric biasing configurations. The resulting phase shift is obtained and plotted in Fig. 4. To obtain the phase shift predicted by the analytical equations, MATLAB has been used to solve for the roots of (7) and (6) and to compute the resulting phase shift for the same set of dimensions and material parameters as those used in the CST model. The results coming from solving the analytical equations are then plotted in the same figure for comparison with those obtained from CST simulations. As can be seen, perfect agreement exists between them for all biased magnetization levels and for both symmetric and anti-symmetric biasing. This verification step gives us confidence in the simulation tool when simulating the following more complex scenario of the complete SIW phase shifter.

It is worth mentioning here that though symmetric biasing yields reciprocal phase characteristics, the maximum available reciprocal phase shift is about three times lower than the nonreciprocal phase shift provided by anti-symmetric bias. This is expected because in symmetric bias the dc static field does not change sign with change of sense of rotation of the RF magnetic field $\left(j H_{x} / H_{z}\right.$ changes sign across $x=a / 2$ for each propagation direction [17], [21]) so instead of strong interaction with one direction of RF propagation and minor interaction with the opposite direction in case of anti-symmetric bias, an average interaction occurs for both directions.

\section{SIW PHASE SHIFTER}

For actual realization, the ferrite LTCC material used in this work is the ESL-40012 tape system provided by electro-science. This material has been characterized for its magneto-static properties in [32]-[34] and has been shown to have a $M_{s}$ in the range from 3000 to $4000 \mathrm{G}\left(f_{m} \approx 8.4-11.2 \mathrm{GHz}\right)$. Differences are probably attributed to the preparation method (firing temperature profile and lamination pressure). Characterization of a sample from our manufacturer (VTT-Electronics) of a ten-layer stack with $145-\mu \mathrm{m}$-thick tape (green tape thickness) revealed that the saturation magnetization is close to $3500 \mathrm{G}\left(f_{m} \approx\right.$ $10 \mathrm{GHz}$ ). This implies that low field losses that occur when the material is partially magnetized could be avoided by operating at frequencies above $10 \mathrm{GHz}$. The values for $\epsilon_{r}$ and $\tan \delta$ were assumed to be 14.8 and $5 \times 10^{-3}$ based on the previous characterization done by [34]. For the magneto-static simulations, the initial $B-H$ curve is required to model the material nonlinearity, the curve from [34] has been utilized as a good approximation.

The designed phase-shifter structure is shown in Fig. 5. It is built in a ten-layer LTCC stack (fired layer thickness is $110 \mu \mathrm{m}$ ) with the SIW occupying the fifth layer. The SIW is 11-mm long with a via center-to-center width of $4.5 \mathrm{~mm}$ to yield a cutoff frequency of about $11 \mathrm{GHz}$. The RF signal is fed through a ground-signal-ground (GSG) launcher that is connected to a microstrip line in layer 5, a tapered transition optimized for operation in the $11.5-13.5-\mathrm{GHz}$ band is then employed to guide the 


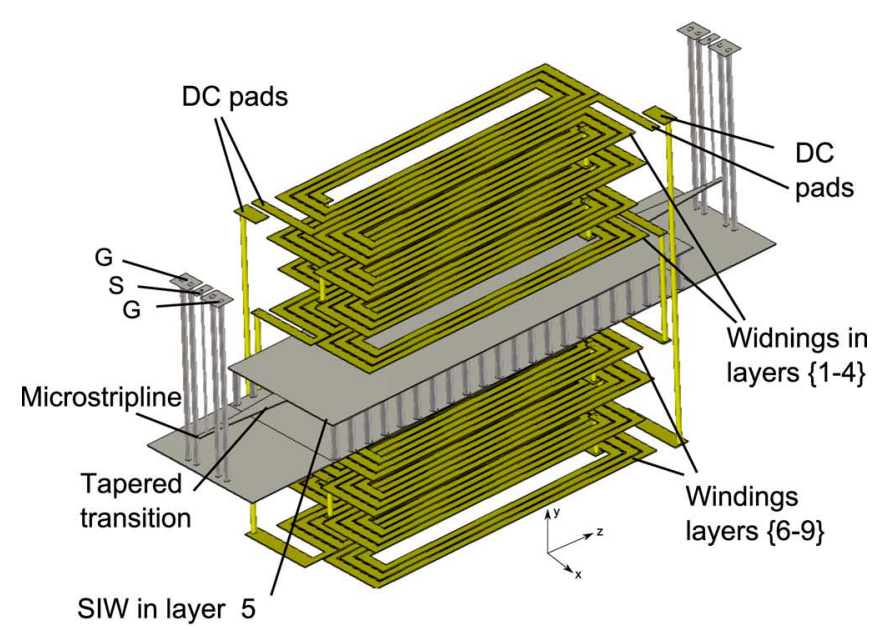

(a)

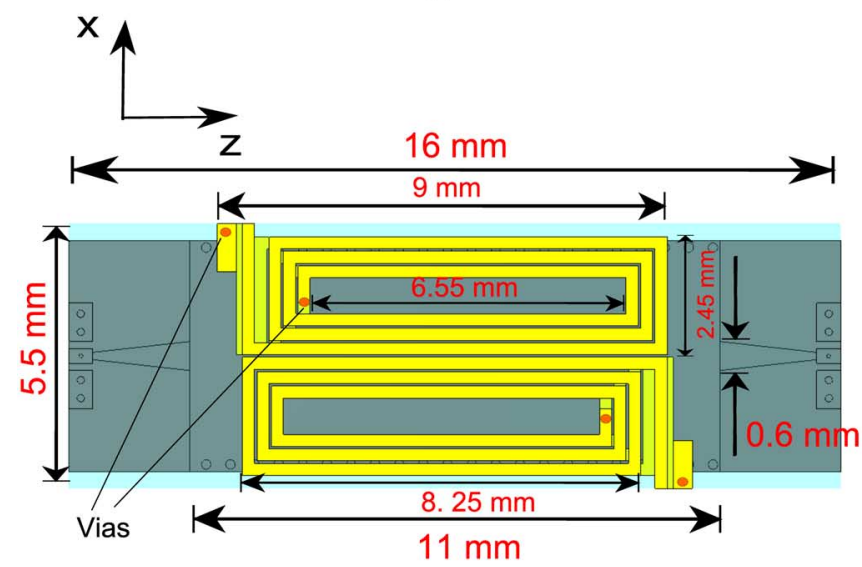

(b)

Fig. 5. SIW phase shifter with feeding structures and embedded bias circuitry. (a) 3-D view. (b) Top view.

wave into the SIW. In order to achieve symmetric and anti-symmetric biasing profiles, an embedded biasing circuit consisting of two solenoid-like coils, placed in odd symmetry about the SIW's central transverse axis, has been integrated with the structure as shown in Fig. 5(b). The coils can be independently driven by currents and thus the direction of the generated $H$ or $M$ by each one can be controlled based on the direction of current flow (clockwise or counter clockwise). Each coil occupies eight layers, with three turns in each layer using $250-\mu \mathrm{m}$-wide traces with $50-\mu \mathrm{m}$ space between turns in the same layer. Vias with $150-\mu \mathrm{m}$ diameter were used to interconnect windings in different layers.

Using CST EM Studio, the magnetization is obtained for each current excitation. A 2-D plot of the generated magnetization profile at the mid-plane of the fifth layer, where the SIW resides, is illustrated in Fig. 6. As can be seen, the profile of the magnetization is generally anti-symmetric in case of opposite current excitation of the coils and symmetric in case of parallel current excitation. An important point to note here is that not all parts of the SIW substrate are getting magnetized to the same level, but the magnetization strength is generally position dependent.

Following the evaluation of the magnetization for each excitation current, the phase shift resulting from each current needs to be obtained by feeding the magnetization generated by that
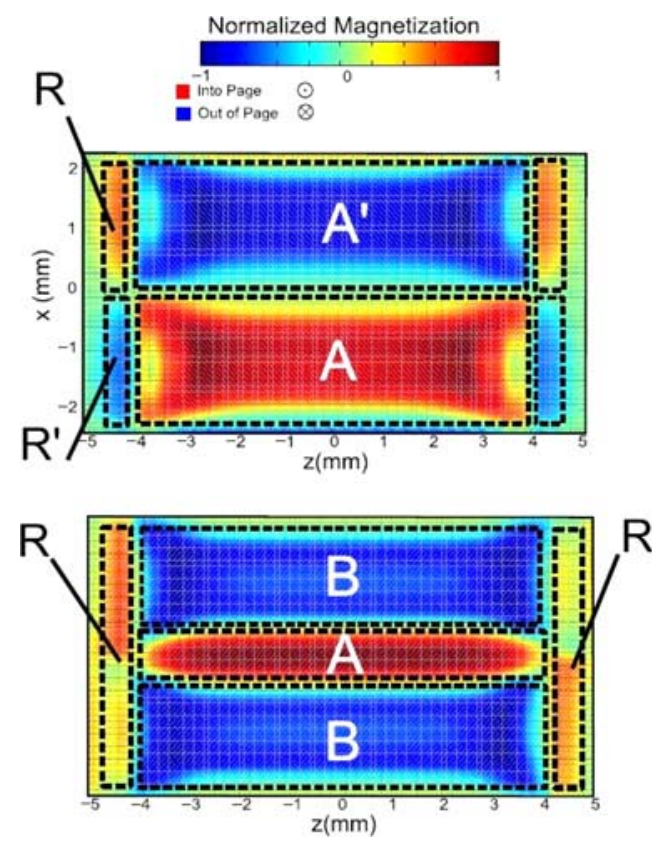

Fig. 6. Simulated magnetization profile at the mid-plane of the fifth layer with anti-parallel current excitation (anti-symmetric bias case) (up) and with parallel current excitation (symmetric bias case) (down) at bias current of $500 \mathrm{~mA}$.

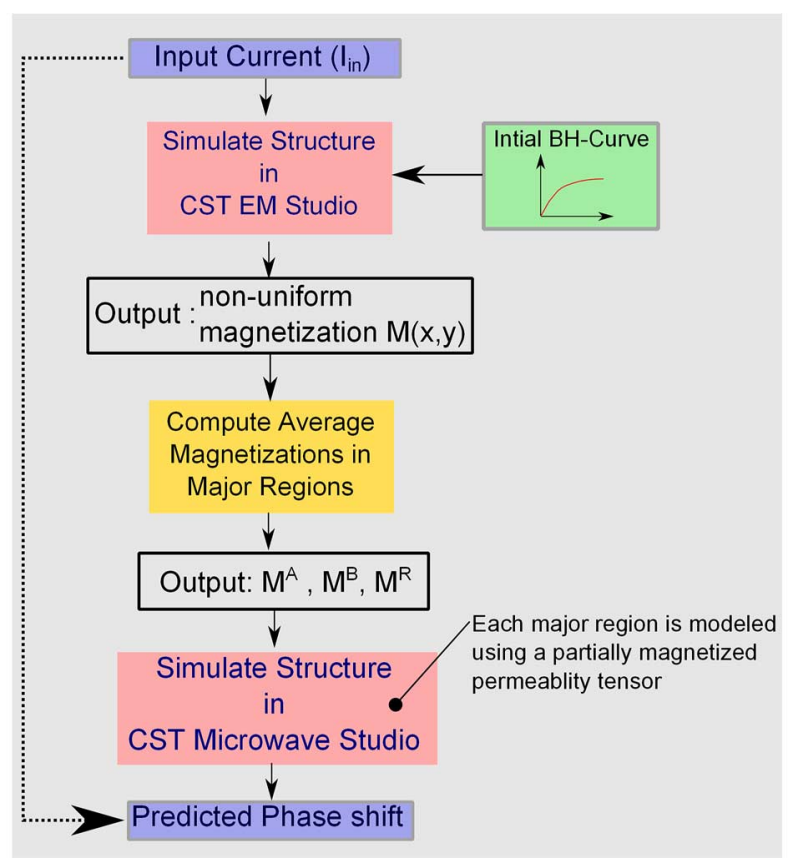

Fig. 7. Steps for prediction of the phase shift resulting from a given input current.

current to CST microwave studio. However, due to the nonuniform nature of the generated magnetization this could not be directly done. This is because the partially magnetized material permeability tensor definition in the microwave simulator is not allowed to have a spatial dependence $[\mu(x, y, z)]_{\mathrm{PMS}}$ and it takes only a single magnetization value per defined volume. To overcome this, we divided the cross section into major regions and modeled each region as a uniformly biased material using the average magnetization calculated for that region. The procedure to obtain the phase shift resulting from a given current is illustrated in Fig. 7. 


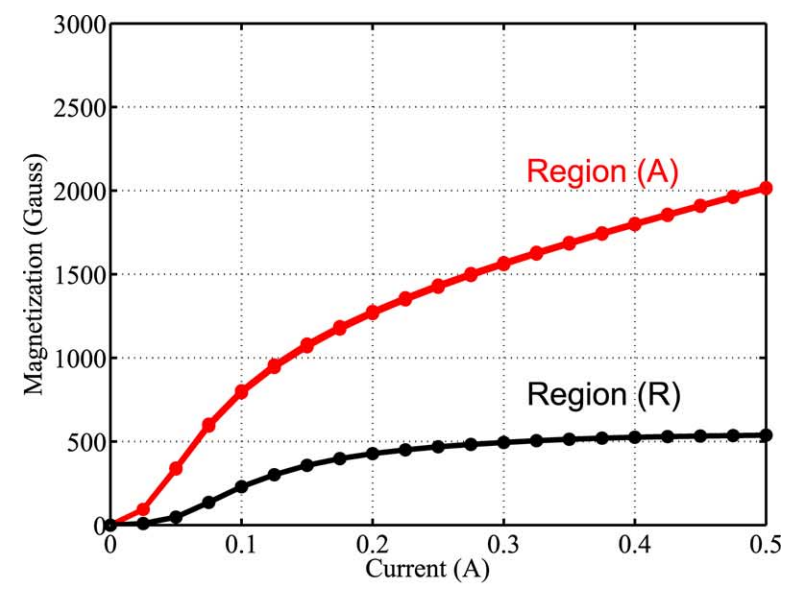

(a)

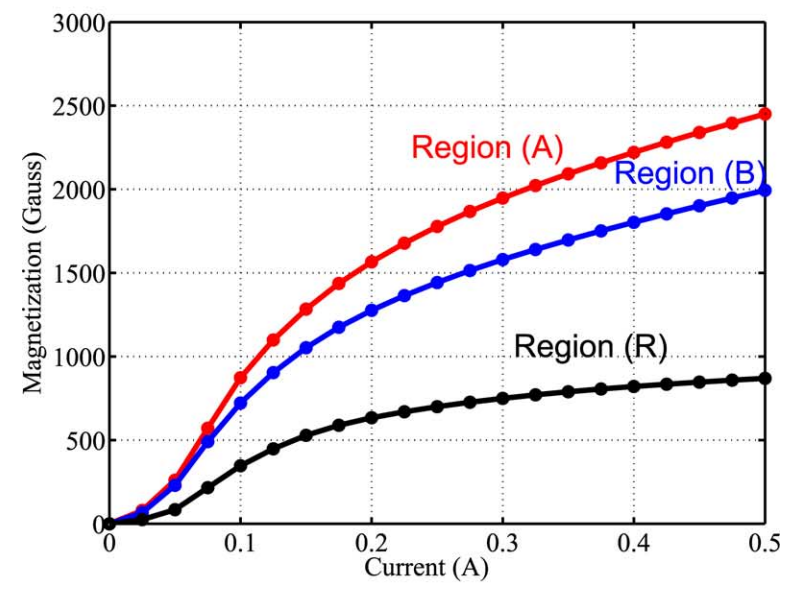

(b)

Fig. 8. Average magnetization for each region shown in Fig. 6 for: (a) antisymmetric and (b) symmetric bias.

In the case of anti-symmetric bias, the material is divided into six major regions, as depicted in Fig. 6, these are: region (A and its image $A^{\prime}$ ) representing the main biased portion of the SIW and the small return of flux path regions $\left(R, R^{\prime}\right)$ that take place towards the coil ends and they nearly extend over a length of about $0.5 \mathrm{~mm}$. Nearly the same has been done in the symmetric case with the exception that the main biased portion of the SIW is divided into three major regions $(\mathrm{B}-\mathrm{A}-\mathrm{B})$, the magnetization level in region $B$ is nearly the same as in region $A$, but in the opposite direction. The average magnetization in each region is plotted in Fig. 8, note that the magnetization in region $\mathrm{A}^{\prime}$ is same as in A, but with a negative sign. Other than the regions defined in Fig. 6, the remaining portion of the substrate is modeled using unbiased material $(\mathrm{M}=0)$. Note that although in the symmetric bias case region $\mathrm{R}$ is not strictly evenly symmetric, as is the case with the main region $(\mathrm{B}-\mathrm{A}-\mathrm{B})$, the whole structure still retains $180^{\circ}$ rotation symmetry about the center point of the plane (origin in Fig. 6) and reciprocity is thus maintained. Under a relatively small bias current of $0.5 \mathrm{~A}$, the magnetization level in the main biased area can reach about $2000 \mathrm{G}$, as shown in Fig. 8. Although increasing the current further should increase the magnetization, and as a result, the phase shift, in practice it is not likely that the printed thin conductor traces $(\approx 3 \mu \mathrm{m})$ can handle reliably currents in excess of $0.5 \mathrm{~A}$. The obtained

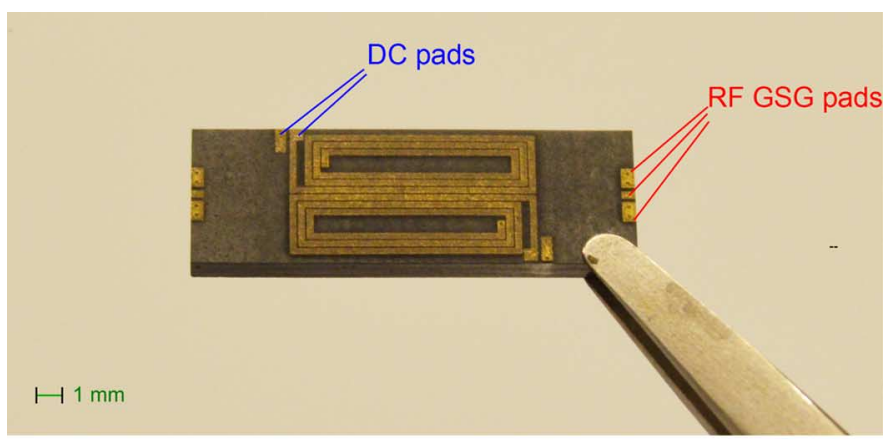

(a)

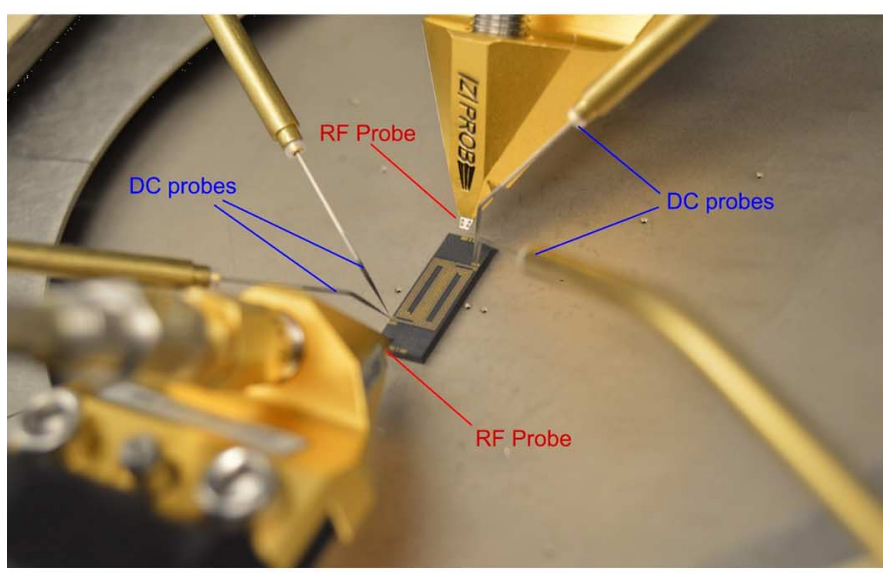

(b)

Fig. 9. (a) Fabricated module and (b) measurement setup.

phase shift for each current, as well as the device S-parameters, are presented in Section $\mathrm{V}$ with their corresponding measured results.

\section{Measurements And Results}

\section{A. S-Parameters Measurements}

The design has been fabricated in VTT's LTCC foundry and the module photograph is shown in Fig. 9(a). Characterization was carried out for the device performance under biased and unbiased conditions using an Agilent PNA series vector network analyzer. The RF signal is launched through coplanar waveguide (CPW) probes contacting the GSG pads on the top layer. For biasing, four dc probes connected to an Agilent E3631 dc power supply have been utilized. The experimental setup is illustrated in Fig. 9(b). First, the S-parameters were measured with no current being applied to any of the windings. The results are illustrated in Fig. 10 with the corresponding simulated ones. As can be seen, a very good agreement exists between them. The device is well matched at both ports over the $11.5-13.5-\mathrm{GHz}$ band, and the insertion loss is better than $3.5 \mathrm{~dB}$ throughout that range, with minimum loss of $2.3 \mathrm{~dB}$ at $12.9 \mathrm{GHz}$.

For phase-shift measurements, the current has been incremented up until $400 \mathrm{~mA}$ in steps of $50 \mathrm{~mA}$ for both bias configurations (symmetric and anti-symmetric) with the S-parameters being recorded each time. The reciprocal phase shift is then calculated as $\left|\angle S_{21}(I)-\angle S_{21}(I=0)\right|$, while the nonreciprocal one is calculated as $\left|\angle S_{21}(I)-\angle S_{12}(I)\right|$. The results for phase shift versus current and frequency under anti-symmetric and symmetric bias are shown in Figs. 11 and 12, respectively. The 


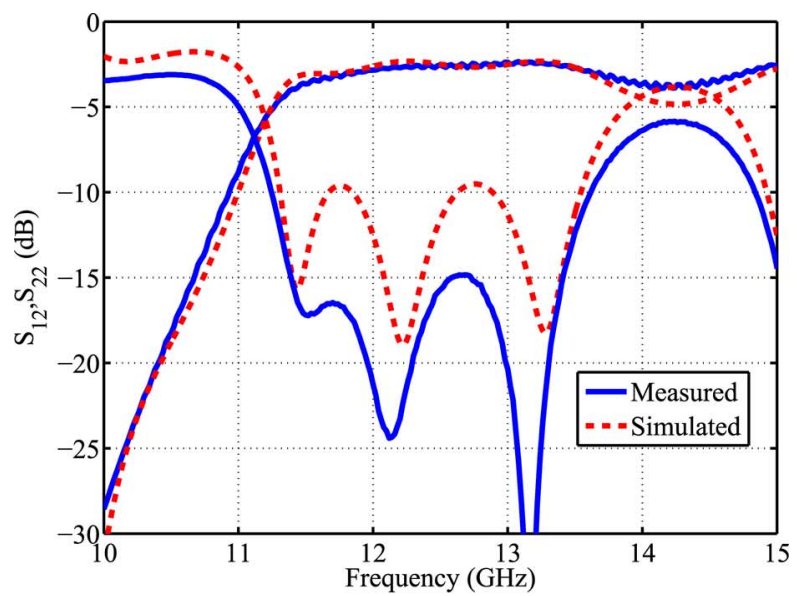

(a)

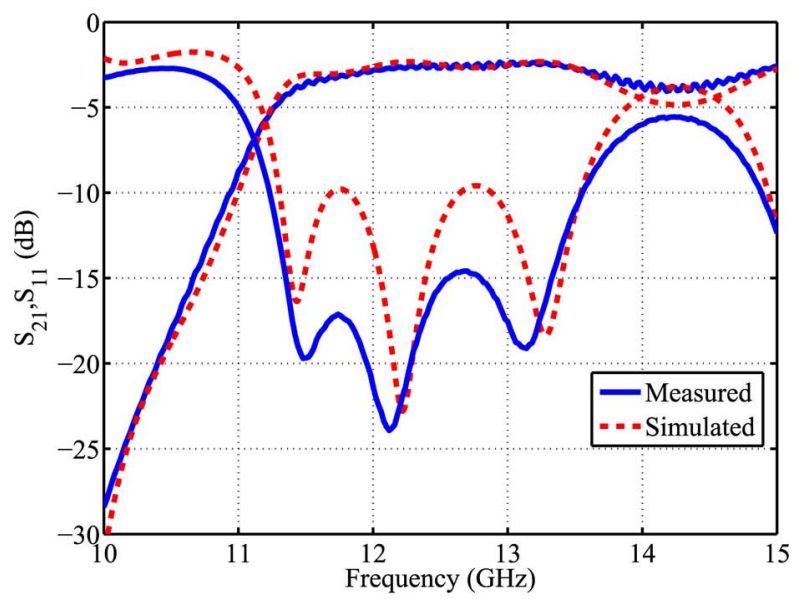

(b)

Fig. 10. Measured and simulated transmission and reflection coefficients at no bias applied.

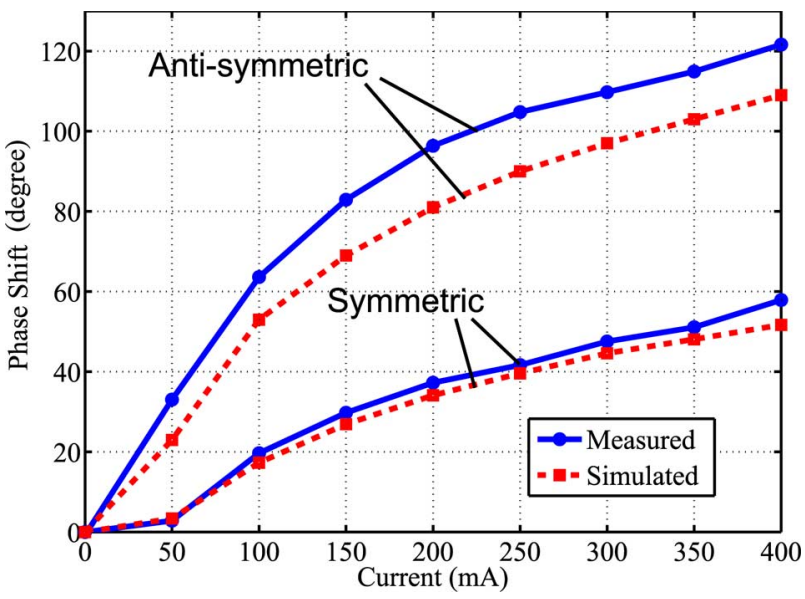

Fig. 11. Simulated and measured phase shift versus bias current at $11.5 \mathrm{GHz}$.

results indicate that the phase shifter achieves a nonreciprocal phase shift of $122^{\circ}\left(153^{\circ} / \mathrm{cm}\right)$ and reciprocal phase shift of $58^{\circ}$ $\left(73^{\circ} / \mathrm{cm}\right)$ at $11.5 \mathrm{GHz}$ with a maximum current of $400 \mathrm{~mA}$.

Also, as can be observed, the measured results agree with the simulated ones to a good extent. In both bias scenarios, the simulation results predict well the trend of phase shift against current and frequency. There is, however, a lean of simulated phase shift to be about $5 \%-10 \%$ lower than the measured ones.

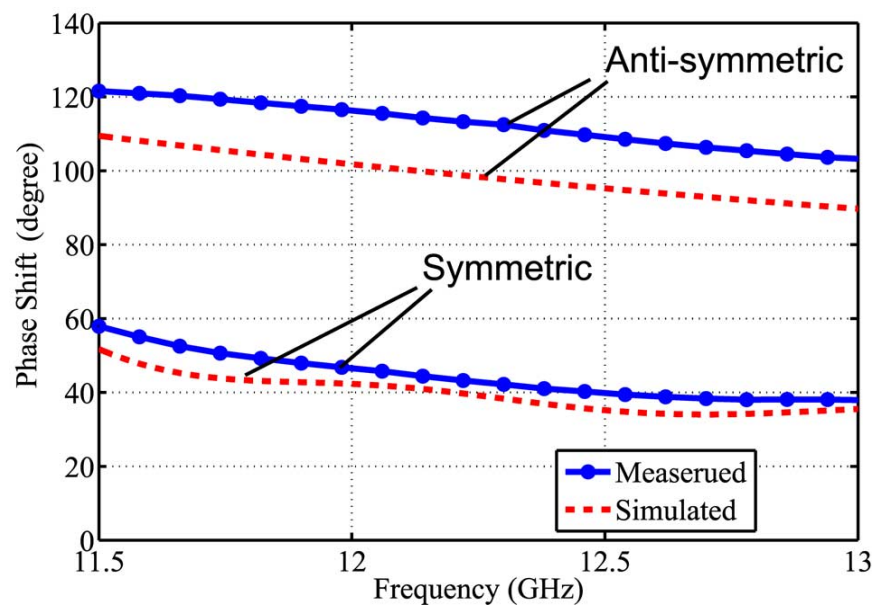

Fig. 12. Simulated and measured phase shift versus frequency at $400 \mathrm{~mA}$ of bias current.

A possible explanation for this might be an inaccuracy of the initial $\mathrm{BH}$-curve used in modeling the material in the simulator, as the measurements indicate that the actual magnetization level is higher than expected for a given current, which may come from underestimation of $B$ originating from a given $H$ in the material's $B H$-curve. Also, the approximation carried out in simulating the biasing profile in the simulator is expected to cause some deviation. Nevertheless, given the multiple factors involved and the problem complexity, we believe the results are satisfactorily well predicted.

For phased antenna array applications, a phase shifter is required to achieve $360^{\circ}$ of tunable phase shift. The large phase shift per unit length demonstrated by this prototype $\left(153^{\circ} / \mathrm{cm}\right.$ at a bias current of $0.4 \mathrm{~A}$ ) implies that a $360^{\circ}$ phase shifter can be easily achieved by using a length of $2.4 \mathrm{~cm}$ while maintaining the same bias current value. Obtaining $360^{\circ}$ in a shorter length is also possible at the cost of increased power/current consumption. By increasing the applied dc current to $1.25 \mathrm{~A}$, simulations indicate that the magnetization level can be driven to saturation $\left(M \rightarrow M_{s}\right)$, which will result in increasing the phase shift per unit length to $220^{\circ} / \mathrm{cm}$. This allows realizing a $360^{\circ}$ phase shifter in a length of $1.7 \mathrm{~cm}\left(360_{\text {degree }} / 220_{\text {degree }} / \mathrm{cm}\right)$. In the fabricated prototype, the bias windings were composed of rather thin and narrow traces, which limited their current-handling capability. As a result, the phase shifter could only be tested with bias current up to $0.4 \mathrm{~A}$.

It is worth mentioning that the measured windings resistance was high $30 \Omega$ (15 $\Omega$ per solenoid) due to using a thin narrow trace for the windings ( $3 \mu \mathrm{m}$ by $250 \mu \mathrm{m}$ ) that resulted in a maximum dc power consumption of about $4.8 \mathrm{~W}$. It is expected, however, that this power can be brought down to about $150 \mathrm{~mW}$ by using thicker and wider windings $(12 \mu \mathrm{m}$ by $500 \mu \mathrm{m})$ and increasing the number of turns per layer and number of layers (six turns/layer for 16 layers) dedicated for the windings

To better judge the performance, the FoM has been calculated for the core SIW section with the exclusion of the feeding losses coming from launchers and transitions (estimated at $1.3 \mathrm{~dB}$ ), which are actually not a part of the phase shifter. The results, depicted in Fig. 13, reveals that the FoM is nearly $100^{\circ} / \mathrm{dB}$ over the $12-13-\mathrm{GHz}$ band for the nonreciprocal case and about 


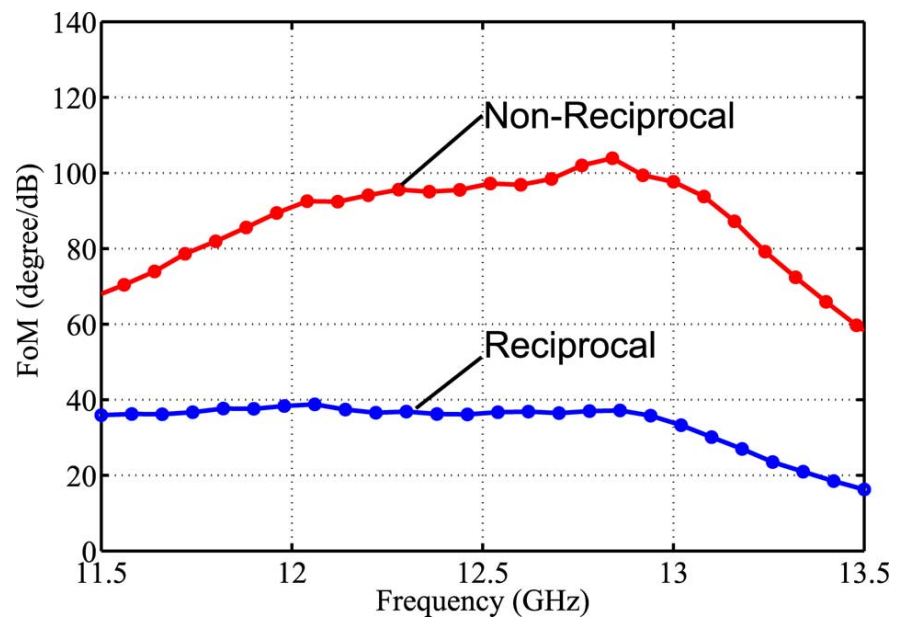

Fig. 13. Phase shifter FoM at $400 \mathrm{~mA}$.

$40^{\circ} / \mathrm{dB}$ for the reciprocal case. These results indicate a significant improvement for this technology.

As can be seen in Fig. 14, the device insertion loss is generally stable over the $12-13-\mathrm{GHz}$ band with less than $0.5 \mathrm{~dB}$ of variation under both biasing scenarios. Also, matching better than $-10 \mathrm{~dB}$ is maintained over the 11.5-13.2-GHz band at both ports, which represent (13.7\%) impedance bandwidth. It worth noting that during the measurements for the reciprocal case, the phase of $S_{12}$ and $S_{21}$ were not exactly identical, as has been the case in simulations. A small frequency and bias varying difference was observed that we refer to as reciprocal phase-shift error $\Delta \theta$. To get a measure of this error, it has been quantified based on its root mean square (rms) value averaged over a frequency band (11-13.5 GHz) and bias (0-400 $\mathrm{mA})$ according to (8), where $N_{I}=9$ and $N_{f}=26$ refer to the number of current and frequency samples, respectively. This error has been found to have a rms value of $1.5^{\circ}$ and a peak value of $4.8^{\circ}$. It is probably coming from small perturbation of the symmetry due to fabrication tolerances and also due to noise in measurements, which was found to cause a $\Delta \hat{\theta}_{\text {Recip }}$ of about $0.5^{\circ}$ for a measured thru standard,

$$
\Delta \hat{\theta}_{\mathrm{Recip}}^{\mathrm{rms}}=\frac{1}{N_{f} N_{I}} \sqrt{\sum_{i=1}^{N_{f}} \sum_{j=1}^{N_{I}}\left|\angle S_{21}\left(f_{i}, I_{j}\right)-\angle S_{12}\left(f_{i}, I_{j}\right)\right|^{2}} .
$$

\section{B. High Power and Nonlinearity Measurements}

In ferrite materials, if the microwave magnetic field strength exceeds a critical value, spinwaves are generated. Spinwaves increase the attenuation of the ferrite material and also give rise to nonlinear effects [26]. Since the microwave signal power is proportional to the square of the magnetic field strength, spinwaves generation sets a maximum microwave power that the device can handle without suffering from performance deterioration. It is thus important to characterize ferrite phase-shifter performance at high power levels. The setup, shown in Fig. 15, has been used to evaluate the device performance (insertion loss and linearity) at high power levels.

For testing the power-handling capability, the input microwave signal power has been swept over the range of -5 to $31 \mathrm{dBm}$. The insertion loss has been measured at $12.4 \mathrm{GHz}$

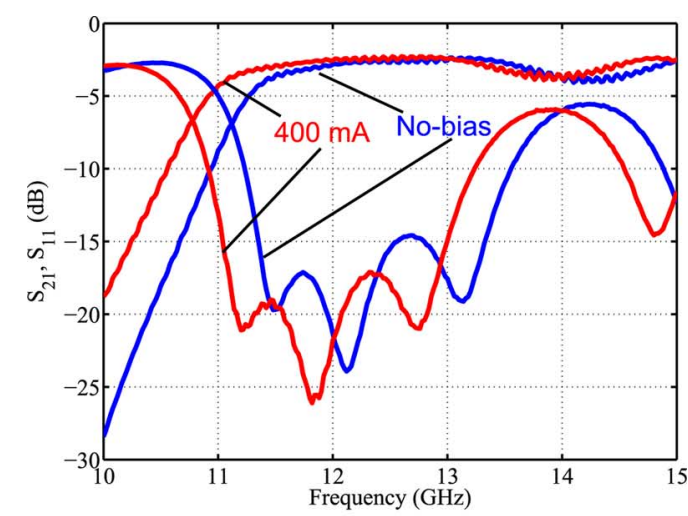

(a)

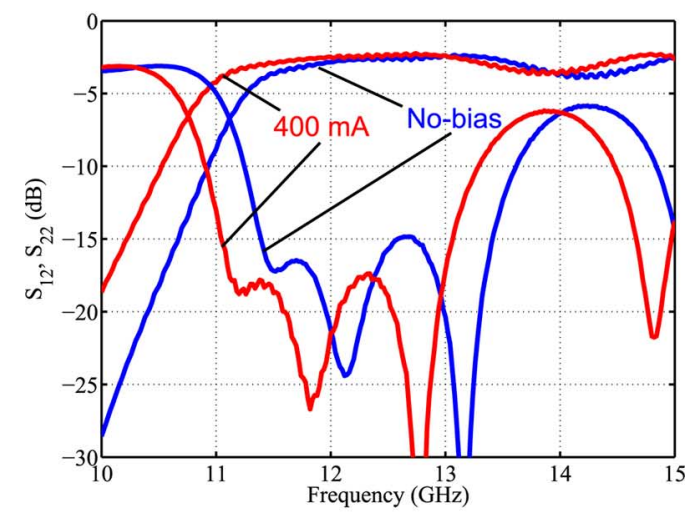

(b)

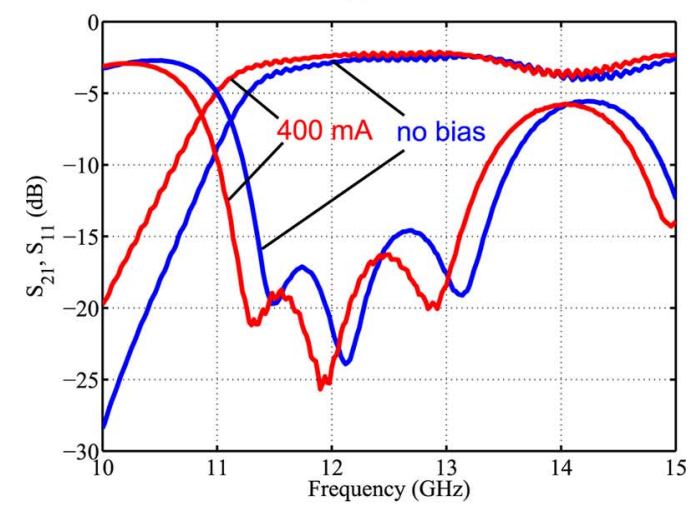

(c)

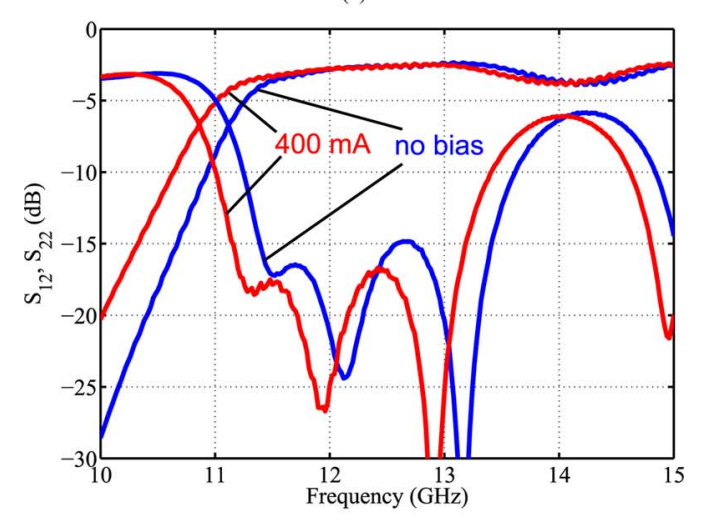

(d)

Fig. 14. Measured transmission and reflection coefficients under: (a) and (b) symmetric bias and (c) and (d) anti-symmetric bias.

under unbiased and biased conditions. The results of the symmetric biasing under different bias current values are depicted in Fig. 16. The results for the anti-symmetric bias are very 


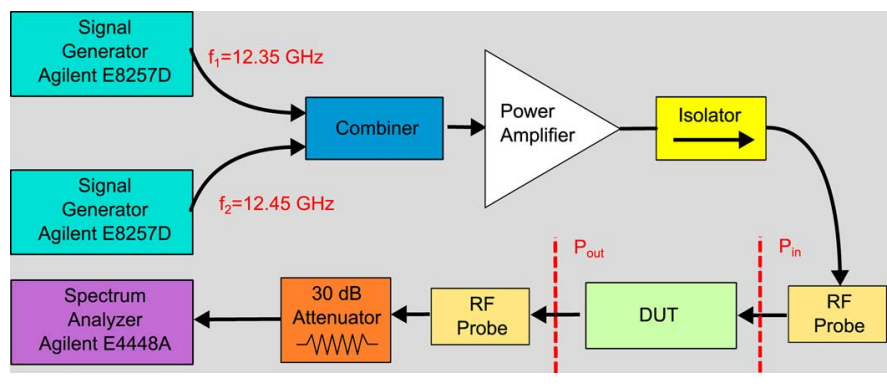

Fig. 15. Block diagram for third-order intercept-point measurements (same setup used for power-handling measurments with a single signal generator connected directly to the power amplifier's input).

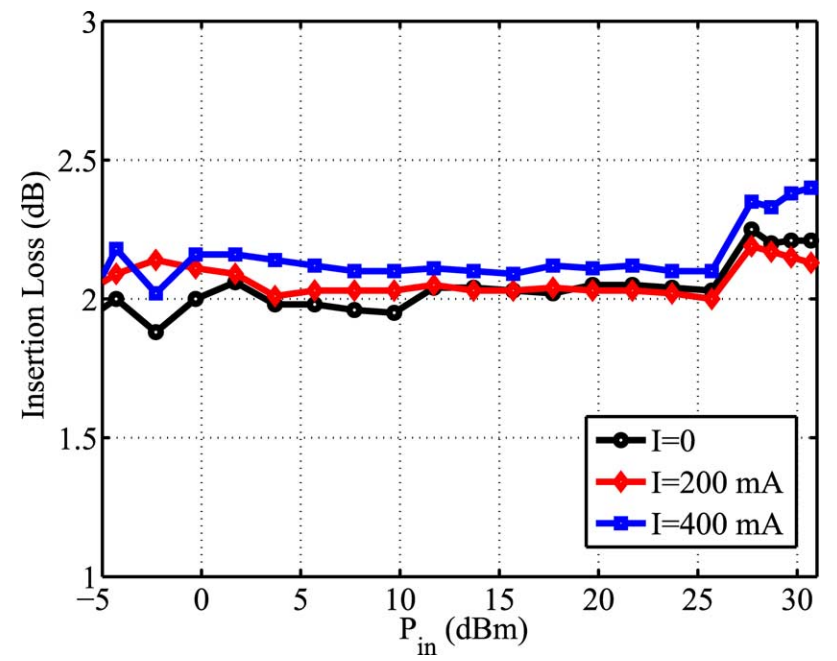

Fig. 16. Measured insertion loss versus input power at $12.4 \mathrm{GHz}$ under symmetric biasing.

similar to the symmetric case. Hence, they are not included here for brevity. As can be seen in Fig. 16, the insertion loss is stable with less than $0.5 \mathrm{~dB}$ of variation up to the maximum input power of our setup $(\approx 1.2 \mathrm{~W})$. This implies that the device can operate at such high power with good performance.

In order to assess the extent of nonlinearity of the device, a two-tone test has been carried out to measure the device's third-order input intercept point $\left(\mathrm{IIP}_{3}\right)$. The block diagram of the measurement setup is demonstrated in Fig. 15. As a first step, a thru standard has been used as a DUT to establish a reference reading. The phase shifter is then placed as the DUT and is characterized. The measurements results, depicted in Fig. 17, indicate an $\mathrm{IIP}_{3}$ of $40.5 \mathrm{dBm}$ for the phase shifter. This value includes not only the nonlinearity of the phase shifter, but also that of the power amplifier. As can be seen from the figure, in the case of a thru standard DUT, there still exists intermodulation products (coming from the power amplifier) that cause an $\mathrm{IIP}_{3}$ of $41 \mathrm{dBm}$. This means that the phase shifter caused only a slight increase in the nonlinearity (compared to the case of the thru), as the IIP ${ }_{3}$ only decreased by $0.5 \mathrm{~dB}$. Therefore, it can be concluded that the device exhibits fairly high linearity with an $\mathrm{IIP}_{3}$ value of at least $40.5 \mathrm{dBm}$.

\section{Comments and Discussion}

Comparison with planar substrate-based ferrite phase shifters existing in the literature is illustrated in Table I. Relative to the prior work employing/using SIW in ferrite LTCC technology in

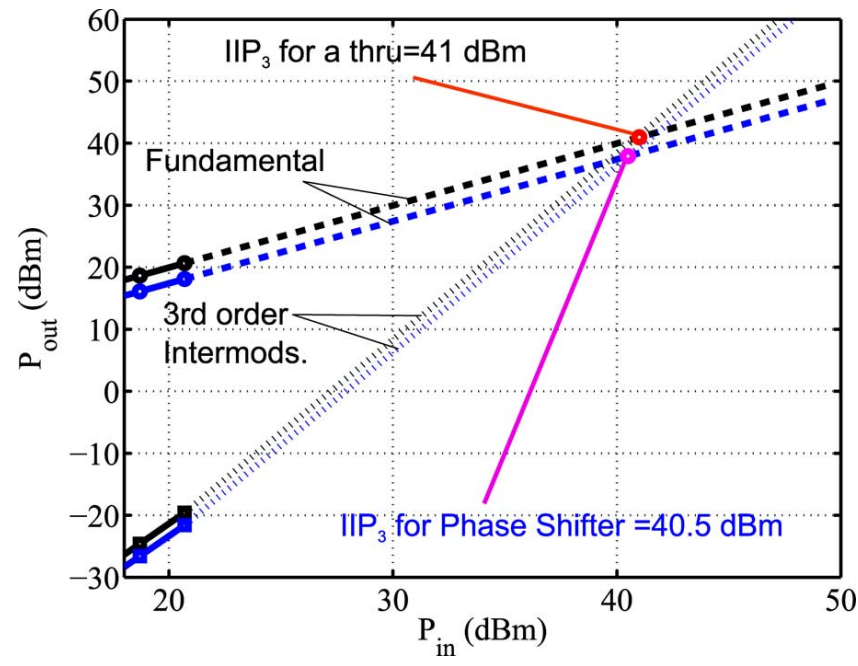

Fig. 17. Measured third-order intercept point (black lines are for a thru as a DUT and blue lines (in online version) are for the phase shifter as a DUT).

[19], it can be observed that we achieved more than five times the phase shift per unit length, higher bandwidth, and overall much better FoM (six times for anti-symmetric bias) and added the capability of providing reciprocal phase shifting. In addition, we used lower maximum current value of $400 \mathrm{~mA}$ compared to $500 \mathrm{~mA}$ used in [19]. The performance of the presented phase shifter even compares well with the one in [13], which used hybrid implementation of a SIW on a machined Duroid substrate that had an inserted ferrite slab. It is important to mention that [13] required huge external magnetic field for tuning of $2400 \mathrm{Oe}$, which is nearly 50 times the magnetic field we require. This huge magnetic field is unlikely to be generated using small coil systems and requires an electromagnet making the overall size of that phase shifter lie in the order of $\mathrm{cm}^{3}$.

The presented phase shifter has a better insertion loss, FoM, and phase shift per unit length relative to microstrip competitors in [23], [24], [35], and [36], which used either coils with magnetic yokes for biasing or electromagnets. Note that in [24] and [35], yttrium-iron-garnet (YIG) substrates were used, which is a known microwave-ferrite material with optimized properties for microwave operation (very low loss, $\tan \delta \approx 10^{-4}$ ) far superior to the non-optimized ESL-40012 ferrite LTCC tape used in this work that has been originally developed as a high-permeability tape for implementing inductors operating at few hundred megahertz.

To give an idea of the size of required coil system for biasing, in [36] a 2000-turn coil was required to produce a 100-Oe field for biasing a $13-\mathrm{GHz}$ phase shifter $\left(8.9 \times 4 \times 0.7 \mathrm{~mm}^{3}\right)$, and it occupied a size of $6 \mathrm{~cm}^{3}$. Also, in [23], a 1000-turn coil system was required to produce a $1000-O e$ field to bias a $3-\mathrm{GHz}$ phase shifter $\left(70 \times 7 \times 5 \mathrm{~mm}^{3}\right)$ that occupied $137 \mathrm{~cm}^{3}$, and even with the use of 1000 turns, a large current of 7.2 A was required. In this work, as a result of using embedded bias winding systems and operating the material in a partially magnetized state, the employed bias magnetic field is on average about $25 \mathrm{Oe}$, which is significantly lower than the bias field used in [13] and [23]. In addition, the coils consist of about 48 turns with a current of $0.4 \mathrm{~A}$ and occupying a volume of about $85 \mathrm{~mm}^{3}\left(0.085 \mathrm{~cm}^{3}\right)$, which shows the huge size saving when compared to the $6-\mathrm{cm}^{3}$ 
TABLE I

Reported Performance of Planar Substrate-Based Ferrite Phase Shifters

\begin{tabular}{|c|c|c|c|c|c|c|c|c|c|c|}
\hline $\begin{array}{l}\text { Ref. } \\
\text { Year }\end{array}$ & $\begin{array}{c}\text { Technology } \\
\text { Ferrite Material }\end{array}$ & $\begin{array}{l}\text { Freq. } \\
\text { (GHz) }\end{array}$ & $\begin{array}{l}\text { BW } \\
(\%)\end{array}$ & Op. & $\begin{array}{l}\text { Phase Shift } \\
\text { (degree) }\end{array}$ & $\begin{array}{l}\text { N. Phase Shift } \\
\text { (degree/cm) }\end{array}$ & $\begin{array}{c}\mathrm{IL} \\
(\mathrm{dB})\end{array}$ & $\begin{array}{c}\text { FoM } \\
\text { (degree/dB) }\end{array}$ & $\begin{array}{c}\text { Biasing } \\
\mathrm{H}(\mathrm{Oe}), \mathrm{I}(\mathrm{A})\end{array}$ & $\operatorname{Size}^{* *}$ \\
\hline $\begin{array}{l}{[23]} \\
2007 \\
\end{array}$ & $\begin{array}{c}\mathrm{MSL} \\
\mathrm{Ni}-\mathrm{Al} \nabla \\
\end{array}$ & 3 & - & - & 700 & 70 & 9 & 78 & $\begin{array}{l}\text { Ext. coil } \\
1000,7.2\end{array}$ & $137 \mathrm{~cm}^{3}$ \\
\hline $\begin{array}{r}{[35]} \\
2009 \\
\end{array}$ & $\begin{array}{l}\text { MSL } \\
\text { YIG }\end{array}$ & 6 & 20 & $\mathrm{R}$ & 100 & 20 & $2.5^{*}$ & 40 & $\begin{array}{l}\text { E.M. } \\
70,-\end{array}$ & $\sim \mathrm{cm}^{3}$ \\
\hline $\begin{array}{r}{[36]} \\
2009\end{array}$ & $\begin{array}{l}\mathrm{MSL} \\
\mathrm{Zn}_{2} \mathrm{Y}\end{array}$ & 13 & - & - & 58 & 65 & 10 & 5.8 & $\begin{array}{l}\text { Ext. coil } \\
100,0.1\end{array}$ & $6 \mathrm{~cm}^{3}$ \\
\hline $\begin{array}{r}{[24]} \\
2013 \\
\end{array}$ & $\begin{array}{r}\mathrm{MSL} \\
\mathrm{YIG}^{\triangle}\end{array}$ & 6.75 & 22 & - & 350 & 36 & $4.3^{*}$ & 72 & $\begin{array}{l}\text { E.M. } \\
100,-\end{array}$ & $\sim \mathrm{cm}^{3}$ \\
\hline $\begin{array}{r}{[19]} \\
2003 \\
\end{array}$ & $\begin{array}{c}\text { SIW } \\
\text { (Ferrite-LTCC tape) }\end{array}$ & 36 & 2.7 & NR & 53 & 26 & 5.8 & 15 & $\begin{array}{l}\text { Emb. coil } \\
<50,0.5\end{array}$ & $175 \mathrm{~mm}^{3}$ \\
\hline $\begin{array}{r}{[13]} \\
2013\end{array}$ & $\begin{array}{c}\text { SIW } \\
\text { Nickel Ferrite }\end{array}$ & 13.25 & 11.3 & NR & 400 & 200 & $2^{*}$ & 200 & $\begin{array}{l}\text { E.M. } \\
2400,-\end{array}$ & $\sim \mathrm{cm}^{3}$ \\
\hline $\begin{array}{l}\text { This } \\
\text { Work }\end{array}$ & $\begin{array}{c}\text { SIW } \\
\text { ESL-40012 } \\
\text { (Ferrite-LTCC tape) }\end{array}$ & 12.4 & 13.7 & $N R, R$ & $\begin{array}{l}N R-R \\
122-58\end{array}$ & $\begin{array}{l}\mathrm{NR}-\mathrm{R} \\
153-73\end{array}$ & 2.3 & $\begin{array}{l}\mathrm{NR}-\mathrm{R} \\
102-39\end{array}$ & $\begin{array}{l}\text { Emb. coils } \\
<50,0.4\end{array}$ & $85 \mathrm{~mm}^{3}$ \\
\hline $\begin{array}{l}\text { Ref. : I } \\
\text { N. phas } \\
\text { FoM ar } \\
*: \text { Indi } \\
\nabla: \text { C- }\end{array}$ & $\begin{array}{l}\text { ference, Freq. : Frequ } \\
\text { shift: Length-normali } \\
\text { IL are based on valu } \\
\text { te use of well establis } \\
\text { A material from Cou }\end{array}$ & $\begin{array}{l}\text { cy, Op. } \\
\text { l phase } \\
\text { at the } \\
\text { micr }\end{array}$ & $\begin{array}{l}\text { perati } \\
\text { hift, B } \\
\text { ximun } \\
\text { ave fe }\end{array}$ & $\begin{array}{l}\text { E.N } \\
\text { Imp } \\
\text { oplie } \\
\text { sub }\end{array}$ & $\begin{array}{l}\text { lectro-magn } \\
\text { ice bandwid } \\
\text { irrent or } \mathrm{H} \\
\text { te, **: Total }\end{array}$ & $\begin{array}{l}\text { R: Reciprocal, } \\
\text { MSL : Microstı } \\
\text { l. Ferrite LTCC } \\
\text { e is calculated }\end{array}$ & $\begin{array}{l}\text { R: N } \\
\text { pline, } \\
\text { based } \\
\text { cludi }\end{array}$ & $\begin{array}{l}\text { eciprocal, Il } \\
\text { b. coil: Emb } \\
\text { ign is highli } \\
\text { biasing circu }\end{array}$ & $\begin{array}{l}\text { Insertion loss. } \\
\text { ded coil, Ext. c } \\
\text { ted in gray. } \\
y,-: \text { unavailabl }\end{array}$ & $\begin{array}{l}\text { External } \\
\text { ry. }\end{array}$ \\
\hline
\end{tabular}

coil used to bias a similar phase shifter at $13 \mathrm{GHz}$. This shows the clear size advantage of the presented phase shifter, as well as its suitability for use in integrated mobile phased arrays.

In addition, the phase shifter, based on preliminary characterization, has been found to handle up to $1.2 \mathrm{~W}$ of input power with no signs of compression of the output and with less than $0.5 \mathrm{~dB}$ of insertion loss variation. It is expected that the device can handle several watts of input power, making it suitable for high-power applications.

\section{CONCLUSION}

In this paper, we have presented a novel SIW phase shifter in ferrite LTCC technology suitable for integration with on-package phased antenna arrays. It is capable of achieving nonreciprocal as well as reciprocal phase shifting by employing anti-symmetric and symmetric bias profiles, respectively. These profiles have been realized by using bias windings embedded within the LTCC package. The phase shifter operates in the partially magnetized state of the ferrite material, and thus requires low magnetic fields for tuning. The fabricated prototype exhibited good phase shift per unit length $(153$ and 73$) \circ / \mathrm{cm}$ and maximum FoM of (102 and 39) $\% \mathrm{~cm}$ for the nonreciprocal and reciprocal phase shifting, which surpasses the previously reported figures using this technology. The presented design is compact, low profile, offers easy integration to antennas, and is mass producible using the LTCC process, making it a good candidate for mobile phased antenna array implementations.

\section{ACKNOWLEDGMENT}

The authors would like to thank K. Kautio and his team at VTT-Electronics, Oulu, Finland, for their efforts in fabricating the prototype.

\section{REFERENCES}

[1] F. Tiezzi, D. Llorens, C. Dominguez, and M. Fajardo, "A compact $\mathrm{Ku}$-band transmit/receive low-profile antenna for broadband mobile satellite communications," in Proc. 4th Eur. Antennas Propag. Conf., Apr. 2010, pp. 1-4.

[2] T. Lambard et al., "Ka-band phased array antenna for high-data-rate SATCOM," IEEE Antennas Wireless Propag. Lett., vol. 11, pp. 256-259, 2012.

[3] C. Boyd, "Ferrite phased array antennas: Toward a more affordable design approach," in IEEE Antennas Propag. Soc. Int. Symp., Jun. 1987, vol. 25 , pp. $1168-1171$.

[4] V. G. Harris, "Modern microwave ferrites," IEEE Trans. Magn., vol. 48, no. 3, pp. 1075-1104, Mar. 2012.

[5] W. E. Hord, "Microwave and millimeter-wave ferrite phase shifters," Microw. J., vol. 32, pp. 81-94, 1989.

[6] K. W. P. A. M. Bozzi and L. Perregrini, "Current and future research trends in substrate integrated waveguide technology," Radioengineering, vol. 18, no. 2, pp. 201-209, 2009.

[7] O. Glubokov and D. Budimir, "Substrate integrated folded-waveguide cross-coupled filter with negative coupling structure," in IEEE Antennas Propag. Soc. Int. Symp., 2009, pp. 1-4.

[8] Z. Hao, W. Hong, J. Chen, H. Zhou, and K. Wu, "Single-layer substrate integrated waveguide directional couplers," Proc. Inst. Elect. Eng.-Microw., Antennas, Propag., vol. 153, no. 5, pp. 426-431, 2006.

[9] Y. J. Cheng, W. Hong, and K. Wu, "Design of a monopulse antenna using a dual v-type linearly tapered slot antenna (dvltsa)," IEEE Trans. Antennas Propag., vol. 56, no. 9, pp. 2903-2909, Sep. 2008.

[10] F. Fesharaki, C. Akyel, and K. Wu, "Broadband substrate integrated waveguide edge-guided mode isolator," Electron. Lett., vol. 49, no. 4, pp. 269-271, Feb. 2013. 
[11] A. Ghiotto, S. Adhikari, and K. Wu, "Ferrite-loaded substrate integrated waveguide switch," IEEE Microw. Compon. Lett., vol. 22, no. 3 , pp. $120-122$, Mar. 2012.

[12] W. D’Orazio, K. Wu, and J. Helszajn, “A substrate integrated waveguide degree-2 circulator," IEEE Microw. Wireless Compon. Lett., vol. 14, no. 5, pp. 207-209, May 2004.

[13] S. Adhikari, A. Ghiotto, S. Hemour, and K. Wu, "Tunable non-reciprocal ferrite loaded SIW phase shifter," in IEEE MTT-S Int. Microw. Symp. Dig., Jun. 2013, pp. 1-3.

[14] A. Shamim, J. Bray, N. Hojjat, and L. Roy, "Ferrite LTCC-based antennas for tunable SoP applications," IEEE Trans. Compon.. Packag.. Manuf. Technol., vol. 1, no. 7, pp. 999-1006, Jul. 2011.

[15] F. Ghaffar, J. Bray, and A. Shamim, "Theory and design of a tunable antenna on a partially magnetized ferrite LTCC substrate," IEEE Trans. Antennas Propag., vol. 62, no. 3, pp. 1238-1245, Mar. 2014.

[16] E. Arabi, F. A. Ghaffar, and A. Shamim, "Tunable bandpass filter based on partially magnetized ferrite LTCC with embedded windings for SoP applications," IEEE Microw. Compon. Wireless Lett., vol. 25, no. 1, pp. 16-18, Jan. 2015.

[17] D. M. Pozar, Microwave Engineering, 3rd ed. New York, NY, USA: Wiley, 2005

[18] S. Stitzer, "Modeling a stripline ferrite phase shifter," in IEEE MTT-S Int. Microw. Symp. Dig., Jun. 1997, vol. 2, pp. 1117-1120.

[19] J. Bray and L. Roy, "Development of a millimeter-wave ferrite-filled antisymmetrically biased rectangular waveguide phase shifter embedded in low-temperature cofired ceramic," IEEE Trans. Microw. Theory Techn., vol. 52, no. 7, pp. 1732-1739, Jul. 2004.

[20] C. Boyd, "A dual-mode latching reciprocal ferrite phase shifter," IEEE Trans. Microw. Theory Techn., vol. MTT-18, no. 12, pp. 1119-1124, Dec. 1970.

[21] S. K. Koul and B. Bhat, Microwave and Millimeter Wave Phase Shifters: Dielectric and Ferrite Phase Shifters. Dedham, MA, USA: Artech House, 1991.

[22] X. Zuo, P. Shi, S. A. Oliver, and C. Vittoria, "Single crystal hexaferrite phase shifter at ka-band," J. Appl. Phys., vol. 91, no. 10, pp. 7622-7624, 2002.

[23] W. Hui, J. Bell, M. Iskander, and J. Lee, "Low-cost microstrip-linebased ferrite phase shifter design for phased array antenna applications," IEEE Antennas Wireless Propag. Lett., vol. 6, pp. 86-89, 2007.

[24] X. Yang et al., "Compact and low loss phase shifter with low bias field using partially magnetized ferrite," IEEE Trans. Magn., vol. 49, no. 7, pp. 3882-3885, Jul. 2013.

[25] R. F. Sooho, Theory and Applications of Ferrites. Englewood Cliffs, NJ, USA: Printice-Hall, 1960.

[26] A. J. B. Fuller, Ferrites at Microwave Frequencies. Stevenage, U.K.: IET, 1986.

[27] D. Bariou, P. Queffelec, P. Gelin, and M. Le Floc'h, "Extension of the effective medium approximation for determination of the permeability tensor of unsaturated polycrystalline ferrites," IEEE Trans. Magn., vol. 37, no. 6, pp. 3885-3891, Nov. 2001.

[28] E. Schlömann, "Microwave behavior of partially magnetized ferrites," J. Appl. Phys., vol. 41, no. 1, pp. 204-214, 1970.

[29] J. J. Green and F. Sandy, "Microwave characterization of partially magnetized ferrites," IEEE Trans. Microw. Theory Techn., vol. MTT-22, no. 6, pp. 641-645, Jun. 1974.

[30] G. T. Rado, "Theory of the microwave permeability tensor and faraday effect in nonsaturated ferromagnetic materials," Phys. Rev., vol. 89, pp. 529-529, Jan. 1953.

[31] P. R. Mcisaac, "Bidirectionality in gyrotropic waveguides," IEEE Trans. Microw. Theory Techn., vol. MTT-24, no. 4, pp. 223-226, Apr. 1976.

[32] P. Fulmek, P. Haumer, I. Atassi, B. Schweighofer, and H. Wegleiter, "Magnetic dc-properties of LTCC-ferrite material and their temperature dependence," IEEE Trans. Magn., vol. 48, no. 4, pp. 1541-1544, Apr. 2012.

[33] W. Zhang et al., "High density integration of high frequency high current point-of-load (POL) modules with planar inductors," IEEE Trans. Power Electron., vol. 30, no. 3, pp. 1421-1431, Mar. 2015.

[34] A. Shamim, J. Bray, N. Hojjat, R. Elasoued, and D. Baillargeat, "Microwave and magnetostatic characterization of ferrite LTCC for tunable and reconfigurable sip applications," in IEEE MTT-S Int. Microw. Symp. Dig., 2007, pp. 691-694.
[35] I. Viswanathan et al., "High performance compact microstripline phase shifter at c-band using yttrium iron garnet," IEEE Trans. Magn., vol. 45 , no. 10 , pp. $4176-4178$, Oct. 2009.

[36] A. L. Geiler et al., "Low bias field hexagonal y-type ferrite phase shifters at ku-band," IEEE Trans. Magn., vol. 45, no. 10, pp. 4179-4182, Oct. 2009.

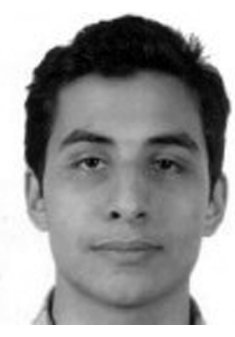

Ahmed Nafe (S'14) was born in Cairo, Egypt, in 1991. He received the B.Sc. degree in electrical engineering (with highest honors) from the German University in Cairo (GUC), Cairo, Egypt, in 2012, and the M.Sc. degree in electrical engineering from King Abdullah University of Science and Technology (KAUST), Thuwal, Jeddah, Saudi Arabia, in 2014.

In 2011, he was a DAAD-sponsored exchange student with Ulm University, Ulm, Germany, where he completed his B.Sc. thesis on design and characterization of millimeter-wave antenna arrays for automotive radars. In the summer of 2011, he joined the Mixed Signal CMOS Circuits group, RWTH Aachen, as a Research Assistant, where he was involved with power amplifier linearization through digital pre-distortion in a multilevel LINC transmitter for WCDMA and long-term evolution (LTE) signals. His current research interests include the analysis and design of reciprocal and nonreciprocal microwave ferrite-based components, microwave and millimeter-wave antennas and arrays, substrate integrated waveguides, and power amplifier linearization techniques.

Mr. Nafe was a recipient of the GUC Scholarship for engineering study in 2008, the KAUST Graduate Student Fellowship in 2012, and the IEEE Magnetics Society Summer School sponsorship in 2015.

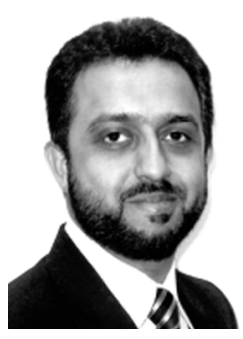

Atif Shamim (SM'13) received the M.A.Sc. and $\mathrm{Ph} . \mathrm{D}$. degrees in electrical engineering from Carleton University, Ottawa, ON, Canada, in 2004 and 2009 , respectively.

From 2007 to 2009, he was an Natural Scientific and Engineering Research Council (NSERC) of Canada Alexander Graham Bell Graduate Scholar with Carleton University. In 2009, he was an NSERC Postdoctoral Fellow with the Royal Military College Canada. In 2010, he joined the Electrical Engineering Program, King Abdullah University of Science and Technology (KAUST), where he is currently an Assistant Professor and Principal Investigator with the IMPACT Laboratory. In 2006, he was an Invited Researcher with the VTT Micro-modules Research Center, Oulu, Finland. He has authored or coauthored over 80 international publications. He holds nine patents. His research interests are integrated on-chip antennas, low-power CMOS RF integrated circuits (RFICs) for system-on-chip (SoC) applications and advanced system-on-package (SoP) designs in multilayer low-temperature co-fired ceramic (LTCC), liquid crystal polymer (LCP), and paper substrates through screen and inkjet printing techniques.

Dr. Shamim serves on the Editorial Board of the IEEE TRANSACTIONS ON ANTENNAS AND PROPAGATION. He is the founding chair of the IEEE Microwave Theory and Techniques Society (IEEE MTT-S) and Antennas and Propagation Society (AP-S) Joint Chapter for Western Saudi Arabia. He was the recipient of the Best Paper Prize at the European Microwave Association Conference in 2008. He was the recipient of the 2008 Ottawa Centre of Research Innovation (OCRI) Researcher of the Year Award in Canada. His research on the wireless dosimeter was bestowed the ITAC SMC Award of Canadian Microelectronics Corporation TEXPO in 2007. He was the recipient of the Best Student Paper Finalist Prize of the IEEE AP-S Conference in 2005. He has also been the recipient of numerous business-related awards, including First Prize in Canada's National Business Plan Competition. He was selected for the OCRI Entrepreneur of the Year Award in 2010. 